\title{
Entangled gene regulatory networks with cooperative expression endow robust adaptive responses to unforeseen environmental changes
}

\author{
Masayo Inoue $\odot^{1}$ and Kunihiko Kaneko $\odot^{2,3}$ \\ ${ }^{1}$ School of Interdisciplinary Mathematical Sciences, Meiji University, Tokyo 164-8525, Japan \\ ${ }^{2}$ Department of Basic Science, Graduate School of Arts and Sciences, University of Tokyo, Tokyo 153-8902, Japan \\ ${ }^{3}$ Center for Complex Systems Biology, Universal Biology Institute, University of Tokyo, Tokyo 113-0033, Japan
}

(Received 3 February 2021; accepted 30 July 2021; published 23 August 2021)

\begin{abstract}
Living organisms must respond to environmental changes. Generally, accurate and rapid responses are provided by simple, unidirectional networks that connect inputs with outputs. Besides accuracy and speed, however, biological responses should also be robust to environmental or intracellular noise and mutations. Furthermore, cells must also respond to unforeseen environmental changes that have not previously been experienced, to avoid extinction prior to the evolutionary rewiring of their networks, which takes numerous generations. To address the question of how cells can make robust adaptation even to unforeseen challenges, we have investigated gene regulatory networks that mutually activate or inhibit, and we have demonstrated that complex entangled networks can make appropriate input-output relationships that satisfy such adaptive responses. Such entangled networks function when the expression of each gene shows sloppy and unreliable responses with low Hill coefficient reactions. To compensate for such sloppiness, several detours in the regulatory network exist. By taking advantage of the averaging over such detours, the network shows a higher robustness to environmental and intracellular noise as well as to mutations in the network, when compared to simple unidirectional circuits. Furthermore, it is demonstrated that the appropriate response to unforeseen environmental changes, allowing for functional outputs, is achieved as many genes exhibit similar dynamic expression responses, irrespective of inputs including unforeseen inputs. The similarity of the responses is statistically confirmed by applying dynamic time warping and dynamic mode decomposition methods. As complex entangled networks are commonly observed in the data in gene regulatory networks whereas global gene expression responses are measured in transcriptome analysis in microbial experiments, the present results give an answer to how cells make adaptive responses and also provide a different design principle for cellular networks.
\end{abstract}

DOI: 10.1103/PhysRevResearch.3.033183

\section{INTRODUCTION}

Living organisms generally respond appropriately to environmental changes by adapting their internal states to the new conditions. In cells, chemical compositions, in particular gene expression levels, are adjusted to adapt to novel environments. Information on the environmental change is transmitted via a signal transduction network to regulate gene expression patterns.

For a cell to make an appropriate response rapidly and accurately, it will be relevant to transfer the input signal to the output expression pattern unidirectionally through a sequence of chemical responses. Indeed, such unidirectional circuits are ubiquitously observed as direct connections or feed-forward networks from the input in the signal transduction networks. How these unidirectional networks achieve appropriate input-output relationships has previously been extensively investigated [1-3].

Published by the American Physical Society under the terms of the Creative Commons Attribution 4.0 International license. Further distribution of this work must maintain attribution to the author(s) and the published article's title, journal citation, and DOI.
Real cellular networks, however, are often much more complex. They include many components and are entangled. Sometimes such unidirectional circuits may be extracted as a part of the complex network, but it is not clear whether they have the same function as has been determined in isolation $[4,5]$. They generally do not function independently, as the networks are entangled. Why cells have adopted such complex networks, however, is not completely understood.

There are further requirements for cellular responses beyond being quick and accurate. For instance, they should be robust to perturbations [6-10], as the environmental conditions surrounding living organisms normally fluctuate [11-13]. Moreover, each process controlling the cellular concentrations of chemicals is generally stochastic, as the number of molecules involved is sometimes not very large [14-16]. In addition to the robustness to fluctuations and noise, there is another postulate, an "adaptive response to an unforeseen challenge (AUC)," as put forward by Braun [17]: Cells must manage to respond to and survive under unforeseen environmental changes that are not expected by the prescribed input-output responses. In particular, even when cells experience a novel environment for which appropriate output responses are not yet prepared, they have to survive to some degree to avoid extinction, as it takes many generations for the appropriate evolutionary changes to occur. 
Responses to unforeseen challenges are thus required without rewiring the gene regulatory network (GRN) via genetic evolution [18-20].

Direct unidirectional networks optimized for given inputoutput relationships are not expected to be robust. Further, it will be difficult to achieve AUCs with them. If this is the case, and if a complex network can have advantages over unidirectional networks, with regard to robustness and AUCs, this may explain why biological networks are so complex and entangled. However, it is not yet evident if a complex network with many degrees of freedom acquires such robustness and AUCs, as they do not always have functional robustness. It is thus necessary to understand which types of complex networks can have a higher capacity for robustness and AUCs than simple unidirectional networks and to uncover design principles for such networks.

Previously we have evolved GRNs to achieve appropriate input-output relationships. In addition to simple direct or feed-forward networks, we have uncovered another type of entangled network consisting of many components, which can generate an appropriate input-output relationship as a cooperative response of many genes [21]. In particular, this cooperative response emerges when each gene expression is sloppy, that is, with a low Hill coefficient as observed in a real GRN [22-25]. Here, we explore whether this class of networks achieves capacity in noise robustness and AUCs higher than that of traditional direct or feed-forward networks.

\section{MODELS}

We adopted a simplified GRN model [8,21,26-30] and evolved the networks so that they make an appropriate response for each given input (environmental condition). A network is composed of $N$ genes as nodes, which are divided into three types: $N_{I}$ input genes receiving external inputs; $N_{O}$ output genes determining the fitness of the cell; and $N_{M}$ middle-layer genes (ML genes) that transmit the input signals to the output genes $\left(N_{I}+N_{O}+N_{M}=N\right)$.

The expression levels of genes are regulated with each other. The regulation from gene $j$ to gene $i$ is represented by the matrix $C_{i j}$ that takes 1 (excitatory), -1 (inhibitory), and 0 (nonexistent). This matrix represents the GRN. Here the input genes $(i)$ do not receive regulation from others (i.e., $C_{i j}=0$ for $\forall j)$ and the output genes $(l)$ do not regulate others (i.e., $C_{j l}=0$ for $\forall j$ ) for simplicity.

We assume that a cell is adapted if, depending on each input, a target output gene shows an appropriate response by regulating gene expression levels properly. The fitness condition under each input that stimulates one of the input genes $k\left(1 \leqslant k \leqslant N_{I}\right)$ is imposed so that corresponding output gene $\left(N-N_{O}+k\right)$ is better to be expressed. We set $N_{I}=N_{O}=5$, $N_{M}=90$, and $N=100$ by assigning the nodes $1, \ldots, N_{I}$ as the input genes and $N-N_{O}+1, \ldots, N$ as the output genes, while leaving others as ML genes.

\section{A. Gene expression dynamics}

By assuming that the synthesis and degradation of mRNA is much faster than protein synthesis, the concentration of mRNA is adiabatically eliminated [31], such that the protein expression level is proportional to the mRNA concentration (gene expression level). Thus, gene expression just means the protein expression itself. The expression level of a gene is regulated through interactions with other proteins, thus constituting a gene regulation network where the expression of each gene can change with time.

Through suitable normalization, the expression level of a gene is represented by the variable $x_{i}=[0,1](i=1, \ldots, N)$, with the maximal expression level scaled to unity. The time evolution of each expression level is given as follows:

$$
\frac{d x_{i}}{d t}=\frac{1}{1+\exp \left[-\beta\left(y_{i}-y_{T}\right)\right]}-x_{i},
$$

where $y_{i}=I_{k} \delta_{i k}+\sum_{j=1}^{N} C_{i j} x_{j}$ is the total signal received by the $i$ th gene with $\delta_{i k}$ as the Kronecker delta for $k=1, \ldots, N_{I}$. $I_{k}$ shows the external input to the $k$ th input gene. $y_{T}$ denotes a constant threshold, and $\beta$ determines the response sensitivity. The second term represents the degradation.

Equation (1) is regarded as a simplified form of the reaction dynamics of a combination of Hill equations that are often adopted in biochemical models and used to capture common salient behaviors. The response sensitivity $\beta$ in Eq. (1) corresponds to the Hill coefficient as $n \sim \beta y_{T}$ [21]. As $\beta$ becomes larger, the first term in Eq. (1) approaches a step function with a threshold $y_{T}$. However, the sensitivity of each expression is weaker than the step function. Here, the Hill coefficient $n$ representing this sensitivity is typically $2-4$ in the gene expression in a cell and this is much weaker than the step function realized for $n($ or $\beta) \rightarrow \infty$ [22-25].

Initially, the expression level of each gene is set to a randomly chosen level between 0 and 1 and evolves according to Eq. (1) with $I_{k}=0\left(k=1, \ldots, N_{I}\right)$, until $x_{i}$ reaches a steady state. Then, the external input on a single input gene is applied at $t=0$, by switching to $I_{l}=I^{*}$ and $I_{j}=0(j \neq l)$. $I^{*}$ is set to 5 , whereas the results are not affected as long as $I^{*} \gg y_{T}$. We assume that all genes in a network have the same $y_{T}$ and $\beta$ values for simplicity.

\section{B. Selection process}

For the evolution process, paths in the regulation matrix $C_{i j}$ were mutated, and $C_{i j}$ was selected according to the following fitness condition: for each given input, one predominant target among the output genes was expressed. Specifically, only the gene $N-N_{O}+k$ should respond upon the application of input $I_{k}\left(k=1, \ldots, N_{I}\right)$, where the response was given as the difference between the final $\left(\overline{x_{i}^{\mathrm{fin}}}\right)$ and initial $\left(\overline{x_{i}^{\mathrm{ini}}}\right)$ expression levels averaged over a time span, respectively, as

$$
\begin{aligned}
& \text { fitness }=\frac{1}{N_{I}} \sum_{k=1}^{N_{I}}\left(\overline{x_{N-N_{O}+k}^{\mathrm{fin}}}-\overline{x_{N-N_{O}+k}^{\mathrm{ini}}}\right. \\
& \left.-\frac{1}{N_{O}-1} \sum_{j=1, j \neq k}^{N_{O}} \overline{x_{N-N_{O}+j}^{\mathrm{fin}}}\right) .
\end{aligned}
$$

The last term represents a punishment term inhibiting responses by nontarget output genes. This punishment term is necessary to prevent the trivial solution in which all output genes respond to any input, rather than a one-to-one response. 
There can be other possibilities for the punishment term, but here we use the above form as one of the simplest ones.

With this fitness function, we evolve the network structure, that is, the regulation matrix $C_{i j}$, by using a simple genetic algorithm. For each generation, 100 networks are prepared, from which the 25 networks with the highest fitness are selected. The maximal fitness value is 1 , where, in an ideal situation, only the corresponding target gene responds by changing from $\overline{x_{i}^{\text {ini }}}=0$ to $\overline{x_{i}^{\text {fin }}}=1$ in accordance with each external input whereas other (incorrect) output genes keep $x_{i}=$ 0 . From each selected network, $100 / 25=4$ mutant networks are generated. The selection process is repeated with these 100 generated networks. By the "mutation," the regulation matrix $C_{i j}$ is slightly modified, whereas the parameters are kept unchanged. In the mutation process, we fix the number of paths and swap the connection $C_{i j}$ with a small mutation rate (one path is mutated on average for every process). Starting from 100 networks randomly connected with a probability of $2.5 \%$ for $C_{i j}= \pm 1$, respectively, networks with high fitness values are obtained after repeating the selection process several (tens of) thousands of times.

\section{RESULTS}

\section{A. Three types of networks}

After the evolution, the highest fitness with one-to-one correspondence between input-target pairs is achieved. As described in Ref. [21], three types of networks evolved depending on the sensitivity $\beta$ (corresponding to the Hill coefficient) of the gene expression dynamics: direct connections (direct type) evolved for large $\beta$ and intermediate $y_{T}$, and feed-forward networks having side paths (FF-network type) evolved for large $\beta$ and large $y_{T}$. In these two types, each gene's response was sensitive (large $\beta$ ) and showed a rapid and accurate behavior. On the other hand, complex entangled networks with cooperative responses of many genes (cooperative type) evolved when each response was sloppy (small $\beta$ ). The three types showed different characteristics in their network structures, and these were most discernible when their core structures were compared. The core structure was obtained by removing paths successively, one-by-one, as long as the corresponding output gene's response for each input was preserved (e.g., maintaining fitness $>0.8$ ) (Fig. 1). The direct and FF-network types adopted unidirectional circuits from the input to the output genes. The cooperative network type, however, involved many genes and its network was entangled. In this case, with the input, a few genes were locally excited, whereas global inhibition by many other genes followed. The characteristic structure of local excitation and global inhibition (LEGI [32]) was common in this cooperative type. Here, it is of note that the evolutionary simulations were carried out to preserve the total path number of the networks, and the redundant paths out of the core structure remained for all types.

\section{B. Quantitative analysis of entanglement}

In order to characterize the entangled networks of the cooperative type, we counted the number of signal transduction routes from an input gene to the corresponding target gene. (a)

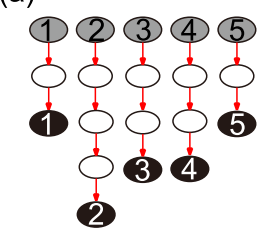

(b)

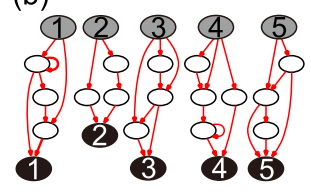

(c)

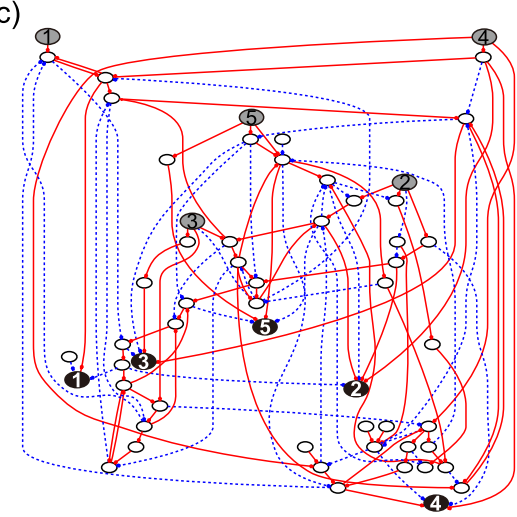

FIG. 1. Typical examples of the three types of core network structures. The core structures were obtained by removing the dispensable paths from the evolved fittest structure for each type. Gray and black circles show the input and output genes, respectively, and the numbers indicate their correspondences. Red bold arrows represent excitatory regulations, and blue dotted arrows denote inhibitory regulations. (a) Direct type with $y_{T}=0.5$ and $\beta=10^{1.75}$, (b) FFnetwork type with $y_{T}=1.0$ and $\beta=10^{1.5}$, and (c) cooperative type with $y_{T}=0.5$ and $\beta=\sqrt{10}$.

Here, we used core structures to exclude surplus routes that were not necessary for the signal transduction, while focusing on the effective routes. Table I shows the average number of routes that were computed as follows. First, we extracted the shortest path(s) connecting an input-target pair. The ML genes on the paths, as well as the input and the target genes, were marked. Then, we extracted the shortest route starting from one of the marked genes and terminating at another of the marked genes. The procedure was repeated until no new routes were possible. Then, the number of such detour routes was counted. As expected, the cooperative type had a much larger number of detours than the other two types of networks and their differences were clearer when compared by their distributions (Fig. 2). The Direct type exclusively showed a sharp peak at one route, whereas the FF-network type with side paths showed smaller peaks around two or three routes. These two types rarely had more than 10 routes. In turn, the entangled cooperative type showed a broad distribution, ranging up to 70 routes. We also measured the number of self-loop structures among ML genes and confirmed that the signal transduction routes in the cooperative type were not

TABLE I. Average number of signal transduction routes.

\begin{tabular}{lllc}
\hline \hline & $y_{T}$ & $\beta$ & Number of routes \\
\hline Direct & 0.5 & 1.75 & 2.03 \\
& 0.75 & 1.75 & 1.12 \\
& 0.25 & 2.0 & 3.50 \\
FF network & 1.0 & 1.5 & 4.42 \\
& 0.75 & 0.75 & 7.40 \\
Cooperative & 1.0 & 1.75 & 3.40 \\
& 0.5 & 0.5 & 39.11 \\
& 0.75 & 0.5 & 27.28 \\
& 0.25 & 0.5 & 59.15 \\
\hline \hline
\end{tabular}




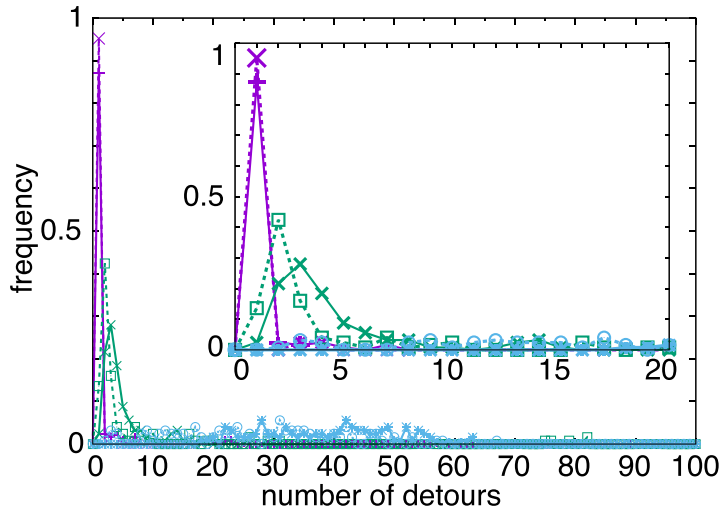

FIG. 2. Distribution of the number of signal transduction routes. The frequency of the number of the routes (abscissa) is plotted for the range of 100 routes. Inset showes the zoom up to 20. From each 5 evolved networks, 5 core structures were obtained and a total of 25 networks were used for each parameter set. Results of the direct type (purple + with solid line when $y_{T}=0.5$ and $\beta=10^{1.75}$, and purple $\times$ with dashed line when $y_{T}=0.75$ and $\left.\beta=10^{1.75}\right)$, the FFnetwork type (green $\times$ with solid line when $y_{T}=1.0$ and $\beta=10^{1.5}$, and green $\square$ with dashed line when $y_{T}=0.75$ and $\beta=10^{0.75}$ ), and the cooperative type (blue $*$ with solid line when $y_{T}=0.5$ and $\beta=$ $10^{0.5}$, and blue $\circ$ with dashed line when $y_{T}=0.75$ and $\beta=10^{0.5}$ ) are shown.

arranged just with many independent parallel paths, but were instead entangled with each other (Supplemental Material, Fig. S1 [33]) [34].

In the following sections, we study and compare the characteristic features of the above three types, with regard to the robustness to noise and mutation and to adaptive response to an unforeseen challenge. We also explain the origin of their differences from the viewpoint of the entangled network structure.

\section{Noise robustness}

Robustness to noise was investigated first. A white Gaussian noise term with amplitude $\xi$ was added to the time evolution of each expression level [Eq. (1)] for the ML genes [Figs. 3(a) and 3(b)] or the stimulated input genes [Fig. 3(c)], and the corresponding Langevin equations were simulated. Here we adopted the Langevin equation to examine the influence of noise. Indeed, chemical Langevin equations are often used to account for the influence of molecular fluctuations, as is also obtained as a continuum counterpart of the stochastic simulation $[35,36]$. Here we added the term $\sqrt{x_{i}} \eta_{i}(t)$ [Fig. 3(a)] or $\eta_{i}(t)$ [Fig. 3(b)] with $\eta_{i}(t)$ as a Gaussian white noise; however, the specific form of noise term does not affect the following results.

When the noise term was added to the ML genes, the direct type was the most fragile and the fitness was drastically reduced by the noise [Figs. 3(a) and 3(b)]. The FF-network and the cooperative types showed noise robustness, as their signal transduction routes were multitracked and noise could be canceled out along the pathways. Furthermore, the cooperative type was more robust to smaller amounts of noise, with $\xi<0.1$. This was because the entangled network enabled the averaging out of noise, not only on each gene at the endpoint of multitrack paths but also on every gene in the network. It is of note that the noise-canceling effect was independent of how many genes affected each output gene. An output gene had interaction paths with 6.1 genes in the direct type, 13.0 genes in the FF-network type, and 11.0 genes in the cooperative type, on average. In addition, the total number of paths in a network was set to be the same for all types. Hence, there was no correlation between the number of paths of interacting genes and noise robustness.

We also studied robustness against noise to an input gene [Fig. 3(c)]. Again, the direct type was largely influenced by the input noise as the noise effect was transmitted directly via the straight paths. The FF-network type was less robust than the direct type. This is because its feed-forward network amplifies the input noise as well as the external signal. The cooperative type had higher robustness to the input noise. Again, the noise could be reduced via the detour paths in the pathways.

Here, the path distance between input and output genes (i.e., the number of intermediate ML genes between them) was irrelevant to the noise robustness. The shortest distance was similar for the three types, and the input genes and the corresponding output genes were connected by one or two ML genes. The noise was reduced not through a long-connected pathway but through multitrack, parallel paths.

Moreover, we studied robustness against extrinsic noise by fluctuating parameter values $\left(y_{T}\right.$ and $\beta$ ) with a long-timescale
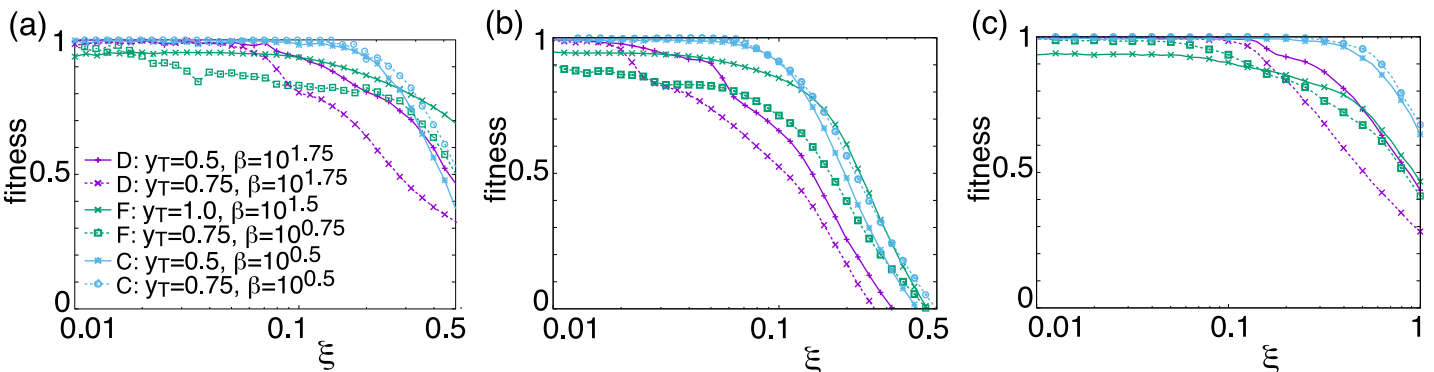

FIG. 3. Noise robustness. Dependence of fitness (ordinate) on noise strength $\xi$ (abscissa). The average fitness values for each noise level are plotted using five different networks that evolved without noise for each parameter set. Noise was added to all ML genes [panels (a) and (b)] or to an input gene stimulated by an external input [panel (c)], and the noise term depended on each expression level [panel (a)] or was independent of it [panels (b) and (c)]. Results of the direct type (D: purple + and $\times$ ), the FF-network type (F: green $\times$ and $\square$ ), and the cooperative type (C: blue $*$ and $\circ$ ) are shown. 


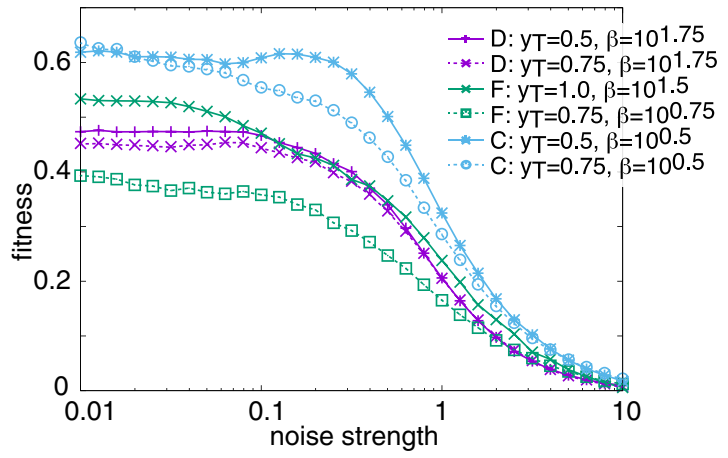

FIG. 4. Robustness against extrinsic noise. The parameters $\zeta\left(y_{T}\right.$ and $\beta$ ) show time change by $d \zeta / d t=-\frac{1}{T}\left(\zeta-\zeta^{*}\right)+\sqrt{\frac{1}{T}} \eta(t)$, with $T=100$, where $\left\langle\eta(t) \eta\left(t^{\prime}\right)\right\rangle=\xi \delta\left(t-t^{\prime}\right)$. Dependence of the fitness (ordinate) on the noise strength $\xi$ (abscissa) is plotted. The $\zeta^{*}$ values are equally selected from parameter sets shown in Table I. Results of the direct type (purple + and $\times$ ), the FF-network type (green $\times$ and $\square$ ), and the cooperative type (blue $*$ and $\circ$ ) are shown using the same networks as in Fig. 3.

$T$ compared with the gene response time (Fig. 4). The parameters show time change according to $d \zeta / d t=-\frac{1}{T}\left(\zeta-\zeta^{*}\right)+$ $\sqrt{\frac{1}{T}} \eta(t)\left(\zeta=y_{T}, \beta\right)$, with $\eta(t)$ being the Gaussian white noise. The direct and FF-network types were fragile by the parameter fluctuations. On the other hand, the cooperative type maintained responses even under large parameter fluctuations by extrinsic noise.

To sum up, the cooperative type was the most robust to both intrinsic and external noise as its entangled network structure contained a larger number of detour paths which showed a noise-canceling effect.

\section{Mutational robustness}

Robustness against the mutational changes in the GRN were then considered. Here, some paths, especially those that constituted a core structure, were inevitable for responses, but some other paths were not necessary to achieve high levels of fitness. The robustness against the removal of the latter was trivial. Thus, mutational robustness should be defined as a fitness change when indispensable paths are removed. Consequently, we studied mutational robustness against the number of removed paths that constituted a core structure (Fig. 5).

The direct type had no mutational robustness, as the response was destroyed when any path that constituted a core structure was removed. The other two network types were more robust against mutational changes as their core structures contained detour paths. Even when a route connecting an input-output pair was disconnected, the external signal could still be transmitted by way of the detour paths. However, the FF-network type and the cooperative type were different in that the core structure of the former was separated according to each input-output pair, but not in the latter. Hence, their mechanisms of mutational robustness differed. In the FF-network type, when a path forming a core structure was removed, the response of a corresponding output gene dropped off, but the responses of the other output genes were hardly

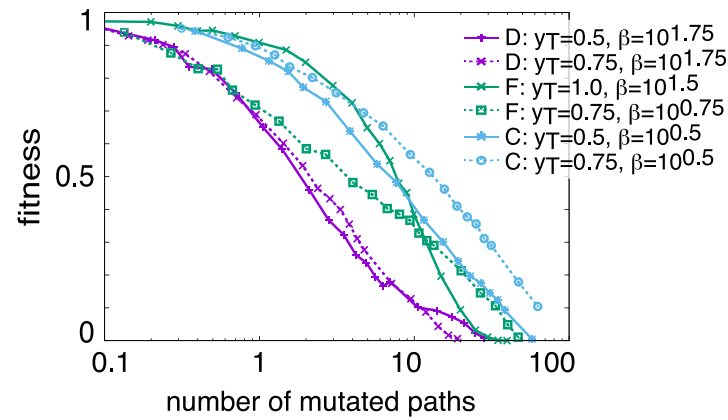

FIG. 5. Mutational robustness. Dependence of the fitness (ordinate) on mutation, i.e., the expected number of removed paths from the core structures (abscissa) is plotted. Results of the direct type (purple + and $\times$ ), the FF-network type (green $\times$ and $\square$ ), and the cooperative type (blue $*$ and $\circ$ ) are shown using the same networks as in Fig. 3.

influenced. In contrast, in the cooperative type with the LEGI structure, the responses of all output genes were weakened when any path in a core structure was removed. However, the decline was smaller as the cooperative type included a larger number of alternative detour paths.

In the evolutionary process so far, we have fixed each parameter (response sensitivity corresponding to the Hill coefficient $\beta$ and a constant threshold for expression $y_{T}$ ) of each gene to a common value and optimized the network structure for the given parameter values. We have also studied the robustness to the changes in these parameter values (Fig. 6). The direct and FF-network types maintained a response only within a parameter range for each type, and the response often disappeared outside of their parameter range. On the other hand, the cooperative type could maintain the response beyond the parameter range corresponding to its phase. This is because the LEGI structure includes the core structures for both the direct and the FF-network types within.

To summarize, the cooperative type showed the highest robustness to both mutations and changes in parameter values. Its entangled network with the LEGI structure contained alternative detour paths and was hardly influenced by mutations. Moreover, the included core structures for the other two types ensure the response over broader parameter ranges.

\section{E. Tolerance to erroneous input}

Thus far, the external input $S_{\text {ext }}$ had been maintained at a steady value for $t \geqslant 0$ in our model. Responses to a pulse input signal that was applied only for a brief time $(0 \leqslant t \leqslant \tau)$ were then investigated (Fig. 7). These signals represent an erroneous input due to environmental fluctuations. It is of note that the timescale for the response dynamics of each gene was set to be scaled to 1 .

The FF-network type was more tolerant than the direct type with simpler motifs. Indeed, the feed-forward motif has been reported to be tolerant to pulse-type inputs [2]. Interestingly, the cooperative type was more tolerant to the pulse input than the FF-network type. In the cooperative type, many ML genes needed to change their expression levels in a sequential order before an output gene responded. An input gene stopped 
(a)

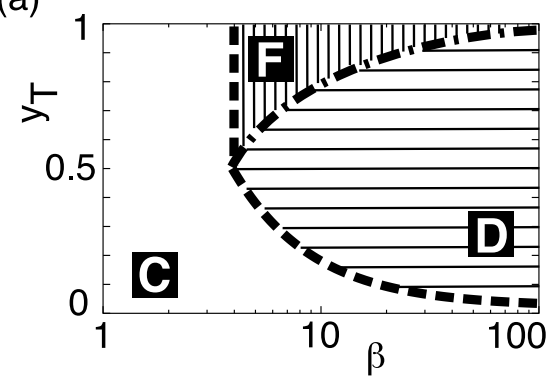

(c)

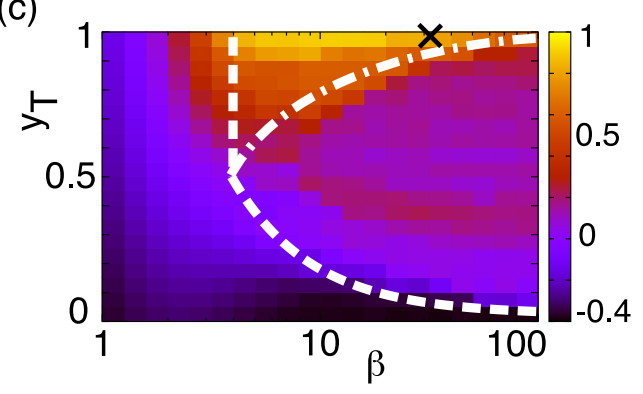

(b)

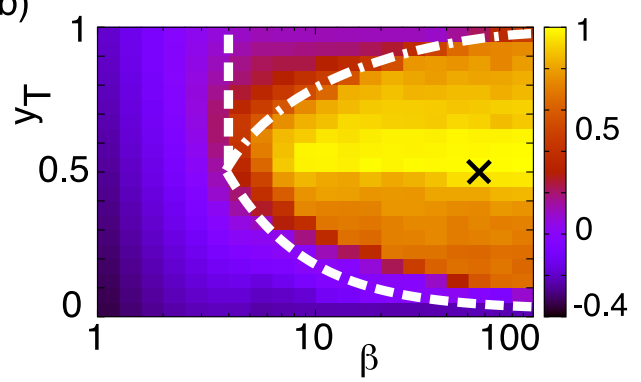

(d)

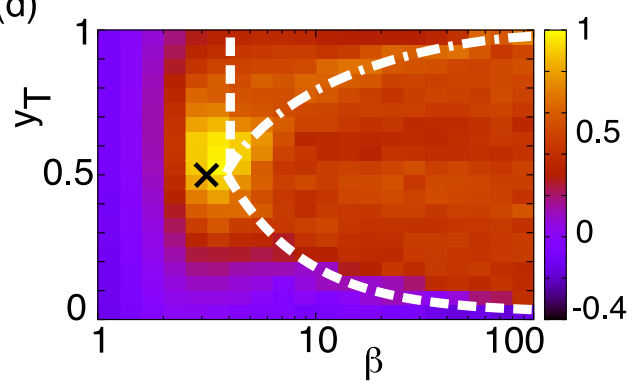

FIG. 6. Robustness to parameter changes. (a) Phase diagram of the three types with regard to the $\beta$ (abscissa) and $y_{T}$ (ordinate) as estimated from the numerical simulations (the phase boundaries were obtained analytically [21]): direct type (D, area with horizontal lines), FF-network type (F, area with vertical lines), and cooperative type (C, blank area). (b-d) Changes in the fitness values against the shift in parameters. By using the network evolved from the parameters denoted by $\times$, fitness was computed for the parameter values $\left(\beta, y_{T}\right)$ in the figure. (b) Direct type with $y_{T}=0.5$ and $\beta=10^{1.75}$, (c) FF-network type with $y_{T}=1.0$ and $\beta=10^{1.5}$, (d) cooperative type with $y_{T}=0.5$ and $\beta=\sqrt{10}$. The white curves indicate the phase boundaries.

responding after a pulse interval, and the following ML genes stopped responding before they stimulated the output gene. Thus, output genes avoided erroneous responses against a pulse input.

\section{F. Adaptive responses to unforeseen challenges}

All living organisms must survive unforeseen environmental changes to avoid extinction. However, evolution is not an easy solution for survival, as it requires the expense of time. If there are no individuals adapted to a new environment

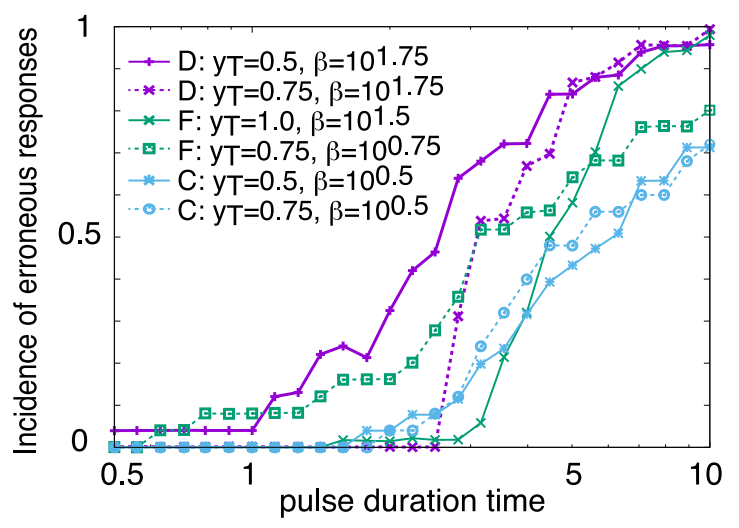

FIG. 7. Incidence of erroneous responses to a pulse input. The occurrence frequency of a response larger than 0.9 upon a pulse input by a corresponding target gene (ordinate) is plotted against the pulse duration time $\tau$ (abscissa). Results of the direct type (purple + and $\times$ ), the FF-network type (green $\times$ and $\square$ ), and the cooperative type (blue $*$ and $\circ$ ) are shown using the same networks as in Fig. 3 . prior to beneficial evolutionary changes, they will go extinct. It is essential for cells to respond to a new environment to some degree without evolution's rewiring of the GRN, and this is referred to as an "adaptive response to an unforeseen challenge (AUC)" [17].

We studied the possibility of the AUCs using the following procedure. We prepared a GRN model by doubling the number of input and output genes but maintained the number of ML genes. Using only half of the input-output pairs, networks were evolved to optimize their fitness, that is, to give postulated output responses to the inputs. Here, half of the input and output genes were not used for the fitness, and hence, they were not related to the original evolution. After the original evolution, we studied the responses of unused output genes when one of the unused input genes was activated. The response to this nonevolutionary input without further changes in the GRN is shown in Fig. 8. Large responses to unforeseen inputs were observed in the cooperative-type networks, whereas there were almost no responses with the FF-network type. The direct type had modest responses; however, this was because its core structure was so simple that even the addition of a random network could generate a response. In addition, such responses were rarer with larger network sizes. Hence, almost no response was expected in a network with many genes.

We also studied how fast a network re-evolves to achieve a response toward an unforeseen change (Fig. 9). The number of mutations required corresponds to the edit distance, which means the number of changes required for a network to achieve the correct response. At first glance, the direct type is most advantageous for re-evolution [Fig. 9(a)]. This is just because its core structure is so simple that a new input-target 

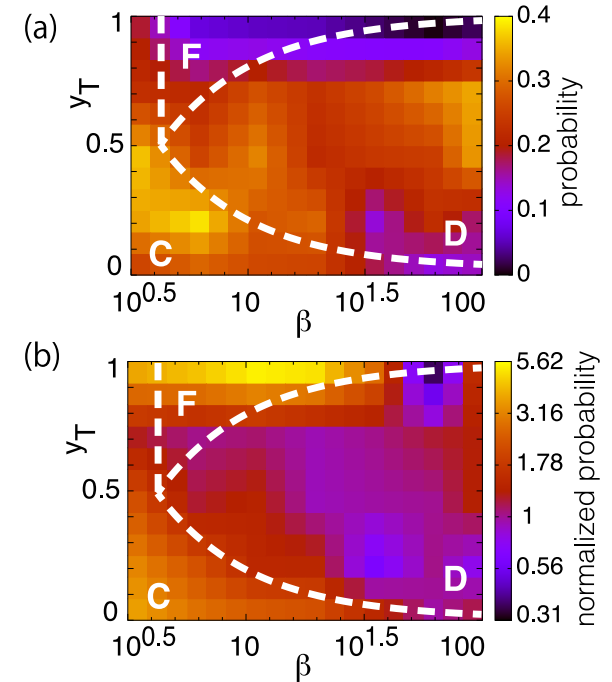

FIG. 8. Responses to an unforeseen input. The response to an unforeseen input was calculated using the fittest networks evolved under both $\beta$ (abscissa) and $y_{T}$ (ordinate) values. The probability that the maximum response was larger than 0.01 (a) and the normalized probability compared with a case of random networks with each parameter value (b) are shown. The white curves show phase boundaries between the three types: direct type (D), FF-network type $(\mathrm{F})$, and cooperative type $(\mathrm{C})$.

pair corresponding to an unforeseen input is often already connected in the redundant paths that existed originally, before the re-evolution process. Therefore, to focus on the effect of mutations, we studied the re-evolutionary process starting from the networks only with the core structures [Fig. 9(b)]. This time, the cooperative type is most rapidly re-evolved toward an unforeseen input. The new network is constructed not from scratch but by utilizing the already evolved structure in the original evolution. On the other hand, in the direct and FF-network types, a new route for a new input needs to be constructed by replacing the original core structures, so that it requires a large number of path changes by mutation.

To sum up, cells that adopt GRNs of the cooperative type can avoid extinction more efficiently as they can show the highest adaptive responses to unforeseen challenges without evolutionary changes as well as within their re-evolutionary process.

\section{G. Resemblance of response dynamics between evolutionary and unforeseen inputs}

One reason why networks of the cooperative type realize the AUC is that many ML genes exhibit similar dynamics in response to the activation of any input gene. They also behave in a similar manner even when an unforeseen input gene that is not used for original evolution is activated. Here, we quantitatively studied the resemblance of the response dynamics of ML genes with different input signals using dynamic time warping (DTW), as shown in Fig. 10 [37-39]. DTW analyzes the similarity between the data of the two time-series, taking into account their elongation and parallel shifts in the time axis direction.
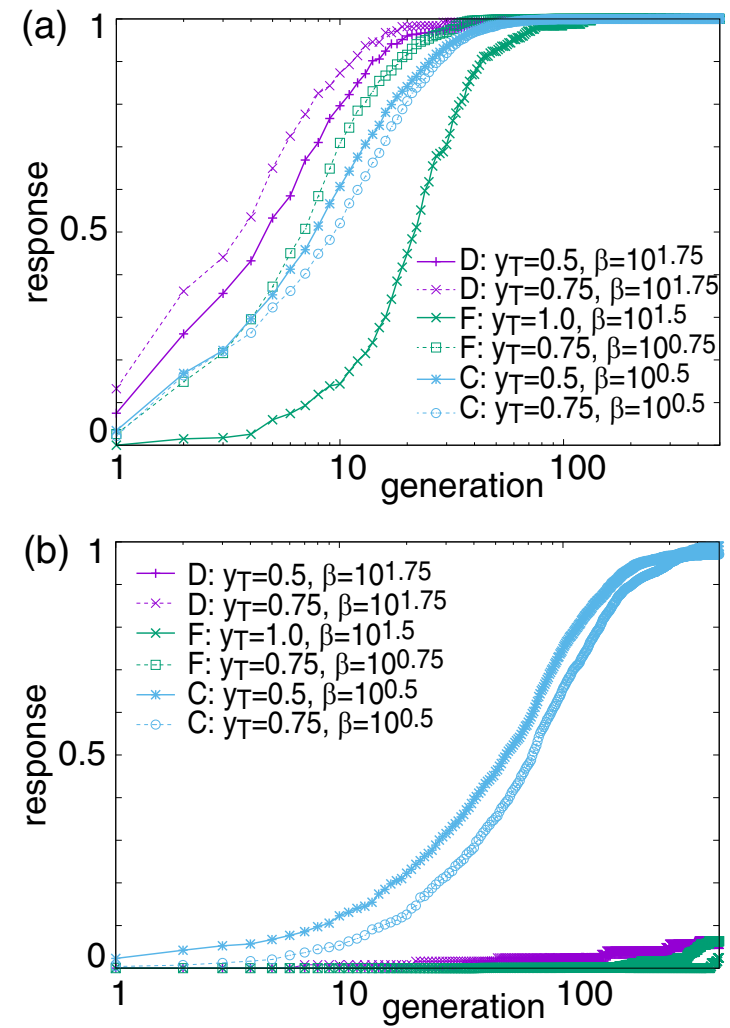

FIG. 9. Time course in re-evolutionary process. Changes in fitness (ordinate) over a re-evolutionary course with an unforeseen input against the generation (abscissa). The networks are evolved to select a larger response to the new target gene corresponding to the unforeseen input. The averaged fitness from 125 different trials is plotted for each parameter set. Networks are re-evolved keeping the number of total paths from the fitted structure after the original evolution (a) or from its core structure (b).

For the cooperative type, the ML-genes' response dynamics to any input, including unforeseen ones, showed remarkable resemblance. These reflected cooperative behavior among the different inputs as well as among the different ML genes. Although each input gene directly activated or inhibited a limited number of ML genes as the network was sparse, almost all ML genes subsequently responded through the entangled network with LEGI; some showed input specific responses, but most genes behaved independently of the input types. For the direct and FF-network types, however, there were almost no such similarities among the different input signals that were observed, and only a small number of ML genes exclusively responded to each input.

Here, it is quantitatively demonstrated that the cooperative expression patterns against different input signals resembled each other. They arise from the entangled structure and enable responses to any input signals including unforeseen ones.

\section{H. Dynamic mode decomposition of response dynamics}

To investigate the origin of the resemblances of the response dynamics more closely, we analyzed the dynamics with dynamic mode decomposition (DMD) [40]. DMD decomposes time-series data with a large degree of freedom into 
(a)

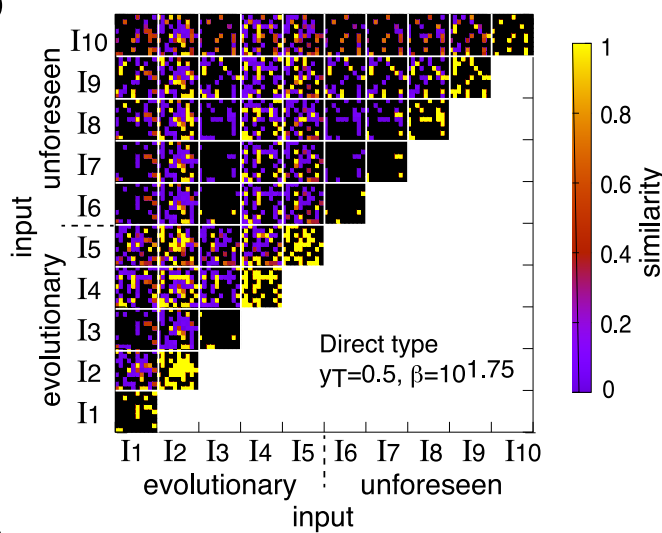

(b)

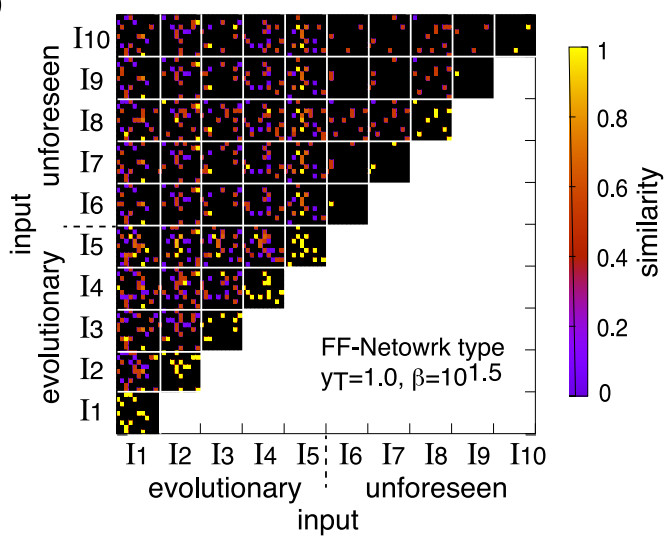

(c)

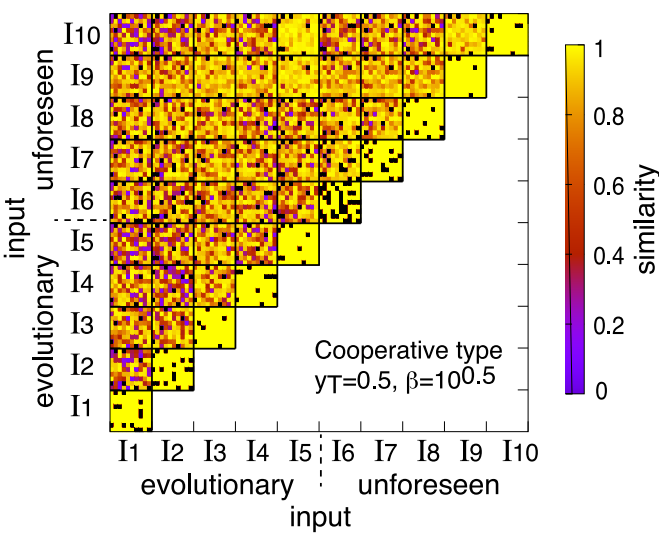

FIG. 10. Similarity in response dynamics against different inputs. Resemblance of the response dynamics of all ML genes with each input signal calculated using dynamic time warping (DTW). Inputs $I_{1}-I_{5}$ are original evolutionary conditions and $I_{6}-I_{10}$ correspond to unforeseen inputs. Each square at the intersection shows the similarity of the ML-gene expression against $I_{i}$ and $I_{j}$. For each square, there are the number of ML-gene pixels and each pixel represents the distance between the ML gene's responses upon $I_{i}$ and $I_{j}$ measured by DTW. Yellow indicates a complete correspondence (i.e., the responses of the gene upon $I_{i}$ and $I_{j}$ agree), purple indicates disagreement, and black indicates the gene shows no response to $I_{i}$ and $I_{j}$. (a) Direct type with $y_{T}=0.5$ and $\beta=10^{1.75}$, (b) FF-network type with $y_{T}=1.0$ and $\beta=10^{1.5}$, and (c) cooperative type with $y_{T}=0.5$ and $\beta=\sqrt{10}$.

a small number of dominant modes [41-45]. It corresponds to a principle component analysis (PCA) for temporal sequence data.
First, from the data series $\left\{\mathbf{x}_{0}, \mathbf{x}_{1}, \ldots, \mathbf{x}_{t}\right\}$, we defined two time-series matrices $X=\left(\mathbf{x}_{0}, \mathbf{x}_{1}, \ldots, \mathbf{x}_{t-1}\right)$ and $Y=$ $\left(\mathbf{x}_{1}, \mathbf{x}_{2}, \ldots, \mathbf{x}_{t}\right)$ and assumed a relationship of $Y=A X$ with an operator $A$. We calculated the eigenvalues and eigenvectors of $A$ by utilizing the singular value decomposition. When the dimension of each time sample $\mathbf{x}_{i}$ was $D_{0}, A$ was a square matrix of size $D_{0}$ and had $D_{0}$ pairs of eigenvalues and eigenvectors by definition. If only a small number $(r)$ of singular values of $X$ had much larger values than others that were close to 0 , the singular value decomposition could be truncated to include only those $r$ modes. The $r$ modes dominantly influenced the dynamics of the data among the $D_{0}$ eigenvectors of $A$. These dominant eigenvectors corresponded to the primary axes in the PCA.

How the number of dominant modes $(r)$ differed according to the network type was investigated first [see Fig. 11(a)]. For the direct type, the number of dominant modes was almost the same as the number of responding ML genes with each input. For the FF-network type, most ML genes showed no responses to the unforeseen inputs, and hence, $r=1$ truncation was just an artifact. In contrast, the cooperative type showed responses that were represented by a smaller number of dominant modes. Its behavior could be described with fewer modes, although a larger number of ML genes responded to each input. This result also indicated that ML genes behaved cooperatively. It is of note that dimension reduction in high-dimensional adaptive states has been observed both in experiments and simulations [46,47], whereas the results of this investigation provided a reduction in the response dynamics.

Finally, the similarity in the dominant modes depending on the different inputs was investigated. With truncated eigenvectors from time-series data for each input, an inner product between eigenvectors for an evolutionary input and for an unforeseen input was calculated and the distribution of the products was computed. This inner product took a larger (smaller) value when the eigenvectors were aligned (orthogonal), that is, when the dominant modes were similar (diverse). As shown in Fig. 11(b), the cooperative type showed the highest similarity, and the FF-network type had the lowest similarity. These results indicate that the response dynamics to an unforeseen input were like those that evolved with an evolutionary input for the cooperative type. In other words, an unforeseen input was transmitted by utilizing an existing channel optimized for evolutionary inputs. Thus, the cooperative type could respond to unforeseen inputs by activating any of the ML genes in the transfer of inputs.

Here again, the resemblance of expression patterns toward evolutionary inputs and those against unforeseen challenges is demonstrated quantitatively by DMD. Cells can respond to unforeseen inputs by utilizing existing redundant transduction routes.

\section{DISCUSSION}

In this paper, we compared the three types of GRNs that exhibit appropriate input-output responses: direct, FF-network, and cooperative types. It was demonstrated that the last type with complex entangled networks showed cooperative responses involving many genes and it was robust to noise, 
(a)

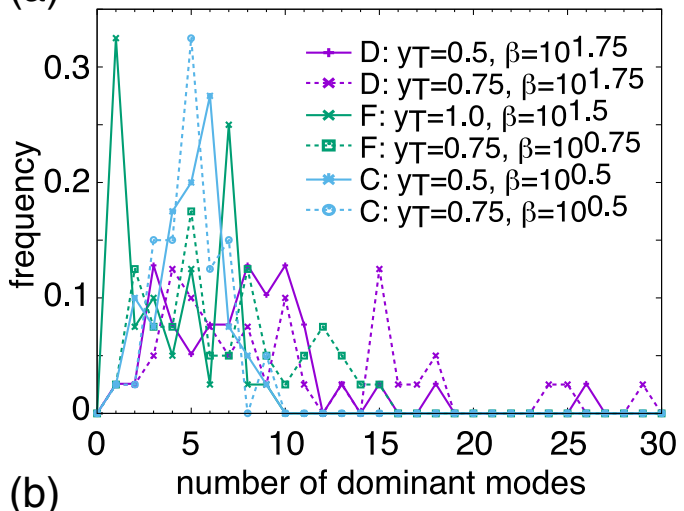

(b)

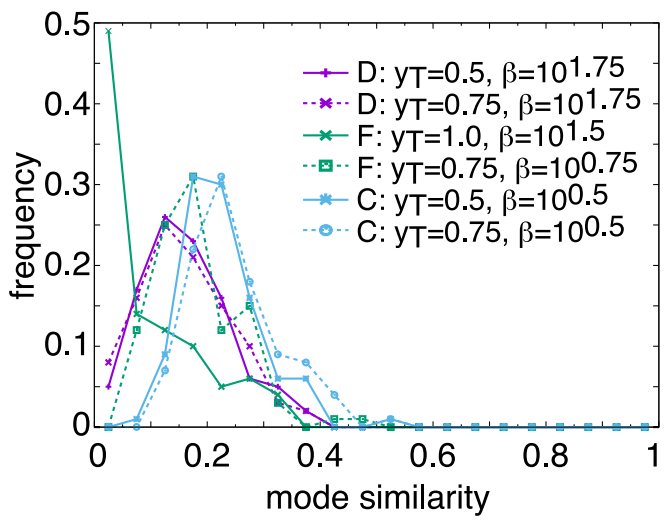

FIG. 11. Response dynamics analyzed with dynamic mode decomposition. (a) Distribution of the number of dominant modes ( $r$; abscissa). $r$ is defined as the number of singular values of a timeseries matrix $X$ that are larger than 1 . Four different fitted networks were used for each parameter set, and ten time-series data sets were computed for each network corresponding to each input, including both evolutionary and unforeseen inputs. (b) Distribution of DMD mode similarity between modes for an evolutionary input and modes for an unforeseen input (abscissa). After computing five dominant DMD modes (eigenvectors) for each input, the mode similarity was obtained as the average value of the inner products between eigenvectors for respective inputs. For the time-series data corresponding to five evolutionary inputs and five unforeseen inputs, that is, $5 \times 5=$ 25 combinations, the DMD mode similarity was computed to obtain the frequency histogram.

errors in the inputs, and network changes. Furthermore, the cooperative type also showed adaptive responses to unforeseen challenges. Hence, this type of network has biological advantages over the others. Even though the other two simple, unidirectional circuits, either direct or feed-forward networks, could show rapid and accurate responses, the entangled network with cooperative responses could better deal with the fluctuating and unforeseen environmental changes.

It is of note that complex networks with cooperative responses emerge when the expression responses of genes are sloppy, in other words, have a low Hill coefficient. This contrasts with the unidirectional simple networks that emerge when the gene responses are sensitive and accurate (with a high Hill coefficient). In general, even after evolution, the Hill coefficients are not very high, and the expression of each gene is sloppy. The cooperative type networks evolved to compensate for the sloppy and unreliable response of each gene. Accurate responses by an ensemble of unreliable sloppy elements were originally proposed by von Neumann for the design of computers. Here, as a byproduct, the present gene expression dynamics were robust to perturbations and showed adaptive responses to unforeseen challenges [48].

The cellular networks are obviously entangled and composed of many elements that interact with each other. Cooperative responses have also been verified experimentally [49-54]. Gasch et al. studied the diverse environmental stress responses of yeast by transcriptome analysis. They reported that certain sets of genes (approximately 900 genes) exhibited similar responses to almost all environmental changes, while some genes showed unique response patterns to specific conditions only [49].

One of the most remarkable properties of cells is their ability to adapt to unforeseen changes in environmental conditions [17-20]. Braun et al. found that yeasts adapted to new environments that they had not previously experienced [17]. This adaptive response occurred due to changes in their gene expression levels neither by means of prepared signaling networks nor by rewiring the networks via evolution. Despite the importance of such adaptive responses, how they are achieved remains elusive. The present investigation indicates a promising solution, by showing that the cooperative responses of the entangled networks induce similar responses for many genes for the experienced and the unforeseen environmental inputs.

As a constructive experiment, Isalan et al. explored the effect of adding genes, i.e., new links to the GRN of E.coli [55,56]. The new gene could provide unforeseen inputs (or perturbations) to the existing GRN and could provide gene expression patterns and outputs similar to those that were accounted for by the existing GRNs. In addition, the gene expression patterns fell into a small number of attractors. This behavior is consistent with the observations for the cooperative type, exhibiting similarity in their responses to unforeseen challenges with those to preexisting inputs.

The origin of the correlated responses for many genes is due to local excitation specific to inputs and the global inhibition of many genes that are not specific to inputs. By considering the latter global response, the adaptive response to unforeseen challenges results. In biological systems, such local-excitation and global-inhibition (LEGI) is often observed, where inhibition occurs globally in space by using the global diffusion of inhibitors, for example, in chemotaxis pathways of eukaryotic cells [57] or Dictyostelium cells $[58,59]$. In this investigation, global inhibition took place not in real space but in network space. We expect that this LEGI behavior can be detected experimentally by global analysis of gene expression patterns and cellular pathways.

Cellular networks with many degrees of freedom are often studied by dividing them into small network motifs [60-62]. It is then assumed that each motif has a characteristic function, whereas the function of the whole network is given as a summation of the functions of the involved motifs. However, paths in the network are entangled, and motifs that work independently are difficult to extract. Moreover, it is not clear 
whether motifs embedded into an entangled network have the same function as in isolation $[4,5]$.

At the same time, we do not necessarily state that the functions of network motifs are completely lost in an entangled network. Indeed, as already explained, the cooperative type is robust against the changes in the parameter values because it includes the core structures of both the direct and FF-network types (Fig. 6). The cooperative type can include such motifs that work in certain situations. In general studies, however, by taking advantage of the entangled network structure, the cooperative response is not decomposed into a combination of motifs.

In summary, the cooperative responses by entangled gene regulatory networks that are robust to noise and mutations can cope with unforeseen challenges, and this is essential for cell survival, as observed in recent microbial experiments.

\section{ACKNOWLEDGMENTS}

The authors thank Macoto Kikuchi, Namiko Mitarai, Naoki Honda, Chikara Furusawa, Nobuhiko J. Suematsu, and Hirokazu Ninomiya for their stimulating discussion. This research was partially supported by a Grant-in-Aid for Scientific Research (A) (Grant No. 20H00123) and a Grantin-Aid for Scientific Research on Innovative Areas (Grant No. 17H06386) from the Ministry of Education, Culture, Sports, Science and Technology (MEXT) of Japan.
[1] S. Mangan and U. Alon, Structure and function of the feedforward loop network motif, Proc. Natl. Acad. Sci. USA 100, 11980 (2003).

[2] U. Alon, An Introduction to Systems Biology: Design Principles of Biological Circuits (Chapman \& Hall/CRC, Boca Raton, FL, 2006).

[3] G. Karlebach and R. Shamir, Modelling and analysis of gene regulatory networks, Nat. Rev. Mol. Cell Biol. 9, 770 (2008).

[4] H.-W. Ma, B. Kumar, U. Ditges, F. Gunzer, J. Buer, and A.-P. Zeng, An extended transcriptional regulatory network of escherichia coli and analysis of its hierarchical structure and network motifs, Nucl. Acids Res. 32, 6643 (2004).

[5] A. Jimenez, J. Cotterell, A. Munteanu, and J. Sharpe, A spectrum of modularity in multi-functional gene circuits, Mol. Syst. Biol. 13, 925 (2017).

[6] K. Kaneko, Life: An Introduction to Complex Systems Biology, Understanding Complex Systems (Springer-Verlag, Berlin, 2006).

[7] S. Ciliberti, O. C. Martin, and A. Wagner, Innovation and robustness in complex regulatory gene networks, Proc. Natl. Acad. Sci. USA 104, 13591 (2007).

[8] K. Kaneko, Evolution of robustness to noise and mutation in gene expression dynamics, PLoS One 2, e434 (2007).

[9] A. Wagner, Robustness and Evolvability in Living Systems (Princeton University, Princeton, NJ, 2007).

[10] S. Nagata and M. Kikuchi, Emergence of cooperative bistability and robustness of gene regulatory networks, PLoS Comput. Biol. 16, e1007969 (2020).

[11] M. B. Elowitz, A. J. Levine, E. D. Siggia, and P. S. Swain, Stochastic gene expression in a single cell, Science 297, 1183 (2002).

[12] C. Furusawa, T. Suzuki, A. Kashiwagi, T. Yomo, and K. Kaneko, Ubiquity of log-normal distributions in intra-cellular reaction dynamics, Biophysics (Biophys. Soc. Jpn.) 1, 25 (2005).

[13] M. Hashimoto, T. Nozoe, H. Nakaoka, R. Okura, S. Akiyoshi, K. Kaneko, E. Kussell, and Y. Wakamoto, Noise-driven growth rate gain in clonal cellular populations, Proc. Natl. Acad. Sci. USA 113, 3251 (2016).

[14] P. S. Swain, M. B. Elowitz, and E. D. Siggia, Intrinsic and extrinsic contributions to stochasticity in gene expression, Proc. Natl. Acad. Sci. USA 99, 12795 (2002).
[15] A. Eldar and M. B. Elowitz, Functional roles for noise in genetic circuits, Nature (London) 467, 167 (2010).

[16] Y. Taniguchi, P. J. Choi, G.-W. Li, H. Chen, M. Babu, J. Hearn, A. Emili, and X. S. Xie, Quantifying E. coli proteome and transcriptome with single-molecule sensitivity in single cells, Science 329, 533 (2010).

[17] E. Braun, The unforeseen challenge: from genotype-tophenotype in cell populations, Rep. Prog. Phys. 78, 036602 (2015).

[18] E. Stolovicki, T. Dror, N. Brenner, and E. Braun, Synthetic gene recruitment reveals adaptive reprogramming of gene regulation in yeast, Genetics 173, 75 (2006).

[19] L. David, E. Stolovicki, E. Haziz, and E. Braun, Inherited adaptation of genome-rewired cells in response to a challenging environment, HFSP J. 4, 131 (2010).

[20] L. David, Y. Ben-Harosh, E. Stolovicki, L. S. Moore, M. Nguyen, R. Tamse, J. Dean, E. Mancera, L. M. Steinmetz, and E. Braun, Multiple genomic changes associated with reorganization of gene regulation and adaptation in yeast, Mol. Biol. Evol. 30, 1514 (2013).

[21] M. Inoue and K. Kaneko, Cooperative reliable response from sloppy gene-expression dynamics, Europhys. Lett. 124, 38002 (2018).

[22] A. Becskei, B. B. Kaufmann, and A. van Oudenaarden, Contributions of low molecule number and chromosomal positioning to stochastic gene expression, Nat. Genet. 37, 937 (2005).

[23] N. Rosenfeld, J. Young, U. Alon, P. Swain, and M. Elowitz, Gene regulation at the single-cell level, Science 307, 1962 (2005).

[24] E. Dekel and U. Alon, Optimality and evolutionary tuning of the expression level of a protein, Nature (London) 436, 588 (2005).

[25] H. D. Kim and E. K. O'Shea, A quantitative model of transcription factor-activated gene expression, Nat. Struct. Mol. Biol. 15, 1192 (2008).

[26] L. Glass and S. Kauffman, The logical analysis of continuous, non-linear biochemical control networks, J. Theor. Biol. 39, 103 (1973).

[27] E. Mjolsness, D. H. Sharp, and J. Reisnitz, A connectionist model of development, J. Theor. Biol. 152, 429 (1991).

[28] I. Salazar-Ciudad, S. Newman, and R. Sole, Phenotypic and dynamical transitions in model genetic networks I. Emergence 
of patterns and genotype-phenotype relationships, Evol. Dev. 3, 84 (2001).

[29] C. Furusawa and K. Kaneko, A generic mechanism for adaptive growth rate regulation, PLoS Comput. Biol. 4, e3 (2008).

[30] M. Inoue and K. Kaneko, Cooperative adaptive responses in gene regulatory networks with many degrees of freedom, PLoS Comput. Biol. 9, e1003001 (2013).

[31] A. Bruce, J. Alexander, W. Peter, L. Julian, R. Martin, and R. Keith, Molecular Biology of the Cell (Garland Science, New York, 2008).

[32] A. Levchenko and P. Iglesias, Models of eukaryotic gradient sensing: Application to chemotaxis of amoebae and neutrophils, Biophys. J. 82, 50 (2002).

[33] See Supplemental Material at http://link.aps.org/supplemental/ 10.1103/PhysRevResearch.3.033183 for details about distribution of the number of loop structures of each middle-layer-gene.

[34] L. Donetti, F. Neri, and M. A. Munoz, Optimal network topologies: Expanders, cages, Ramanujan graphs, entangled networks and all that, J. Stat. Mech.: Theory Exp. (2006) P08007.

[35] D. T. Gillespie, The chemical Langevin equation, J. Chem. Phys. 113, 297 (2000).

[36] N. G. van Kampen, Stochastic Processes in Physics and Chemistry, 3rd ed. (North-Holland, Amsterdam, 2007).

[37] J. B. Donald and C. James, Using dynamic time warping to find patterns in time series, in AAAIWS'94: Proceedings of the 3rd International Conference on Knowledge Discovery and Data Mining (AAAI, California, USA, 1994), p. 359.

[38] J. K. Eamonn and J. P. Michael, Derivative dynamic time warping, in Proceedings of the SIAM International Conference on Data Mining (2001), https://epubs.siam.org/doi/pdf/10.1137/1. 9781611972719.1.

[39] E. Keogh and C. A. Ratanamahatana, Exact indexing of dynamic time warping, Knowl. Inf. Syst. 7, 358 (2005).

[40] P. J. Schmid, Dynamic mode decomposition of numerical and experimental data, J. Fluid Mech. 656, 5 (2010).

[41] C. W. Rowley, I. Mezic, S. Bagheri, P. Schlatter, and D. S. Henningson, Spectral analysis of nonlinear flows, J. Fluid Mech. 641, 115 (2009).

[42] M. Budisic, R. Mohr, and I. Mezic, Applied Koopmanism, Chaos 22, 047510 (2012).

[43] I. Mezic, Analysis of fluid flows via spectral properties of the Koopman operator, Annu. Rev. Fluid Mech. 45, 357 (2013).

[44] J. H. Tu, C. W. Rowley, D. M. Luchtenburg, S. L. Brunton, and J. N. Kutz, On dynamic mode decomposition: Theory and applications, J. Comput. Dyn. 1, 391 (2014).

[45] S. L. Brunton, J. L. Proctor, and J. N. Kutz, Discovering governing equations from data by sparse identification of nonlinear dynamical systems, Proc. Natl. Acad. Sci. USA 113, 3932 (2016).

[46] K. Kaneko, C. Furusawa, and T. Yomo, Universal Relationship in Gene-Expression Changes for Cells in Steady-Growth State, Phys. Rev. X 5, 011014 (2015).

[47] C. Furusawa and K. Kaneko, Formation of dominant mode by evolution in biological systems, Phys. Rev. E 97, 042410 (2018).
[48] J. von Neumann, Probabilistic logics and the synthesis of reliable organisms from unreliable components, Automata Stud. 34, 43 (1956).

[49] A. Gasch, P. Spellman, C. Kao, O. Carmel-Harel, M. Eisen, G. Storz, D. Botstein, and P. Brown, Genomic expression programs in the response of yeast cells to environmental changes, Mol. Biol. Cell 11, 4241 (2000).

[50] H. C. Causton, B. Ren, S. S. Koh, C. T. Harbison, E. Kanin, E. G. Jennings, T. I. Lee, H. L. True, E. S. Lander, and R. A. Young, Remodeling of yeast genome expression in response to environmental changes, Mol. Biol. Cell 12, 323 (2001).

[51] S. Stern, T. Dror, E. Stolovicki, N. Brenner, and E. Braun, Genome-wide transcriptional plasticity underlies cellular adaptation to novel challenge, Mol. Syst. Biol. 3, 106 (2007).

[52] S. Jozefczuk, S. Klie, G. Catchpole, J. Szymanski, A. CuadrosInostroza, D. Steinhauser, J. Selbig, and L. Willmitzer, Metabolomic and transcriptomic stress response of Escherichia coli, Mol. Syst. Biol. 6, 364 (2010).

[53] M. V. Gerashchenko, A. V. Lobanov, and V. N. Gladyshev, Genome-wide ribosome profiling reveals complex translational regulation in response to oxidative stress, Proc. Natl. Acad. Sci. USA 109, 17394 (2012).

[54] Y.-H. Ho, E. Shishkova, J. Hose, J. J. Coon, and A. P. Gasch, Decoupling yeast cell division and stress defense implicates mRNA repression in translational reallocation during stress, Curr. Biol. 28, 2673 (2018).

[55] M. Isalan, C. Lemerle, K. Michalodimitrakis, C. Horn, P. Beltrao, E. Raineri, M. Garriga-Canut, and L. Serrano, Evolvability and hierarchy in rewired bacterial gene networks, Nature (London) 452, 840 (2008).

[56] R. Baumstark, S. Hanzelmann, S. Tsuru, Y. Schaerli, M. Francesconi, F. M. Mancuso, R. Castelo, and M. Isalan, The propagation of perturbations in rewired bacterial gene networks, Nat. Commun. 6, 10105 (2015).

[57] K. Takeda, D. Shao, M. Adler, P. G. Charest, W. F. Loomis, H. Levine, A. Groisman, W.-J. Rappel, and R. A. Firtel, Incoherent feedforward control governs adaptation of activated Ras in a eukaryotic chemotaxis pathway, Sci. Signal. 5, ra2 (2012).

[58] C. J. Wang, A. Bergmann, B. Lin, K. Kim, and A. Levchenko, Diverse sensitivity thresholds in dynamic signaling responses by social amoebae, Sci. Signaling 5, ra17 (2012).

[59] A. Nakajima, S. Ishihara, D. Imoto, and S. Sawai, Rectified directional sensing in long-range cell migration, Nat. Commun. 5, 5367 (2014).

[60] R. Milo, S. Shen-Orr, S. Itzkovitz, N. Kashtan, D. Chklovskii, and U. Alon, Network motifs: Simple building blocks of complex networks, Science 298, 824 (2002).

[61] S. Shen-Orr, R. Milo, S. Mangan, and U. Alon, Network motifs in the transcriptional regulation network of Escherichia coli, Nat. Genet. 31, 64 (2002).

[62] W. Ma, A. Trusina, H. El-Samad, W. A. Lim, and C. Tang, Defining network topologies that can achieve biochemical adaptation, Cell 138, 760 (2009). 